\title{
Cognitive Functioning in Adolescents with Self-Reported ADHD and Depression: Results from a Population-Based Study
}

\author{
Arunima Roy $^{1,2}$ - Albertine J. Oldehinkel ${ }^{1}$. Catharina A. Hartman ${ }^{1}$ \\ Published online: 3 May 2016 \\ (C) The Author(s) 2016. This article is published with open access at Springerlink.com
}

\begin{abstract}
This study aims to assess cognitive functioning differences among adolescents with retrospectively self-reported: ADHD and an onset of depression, only ADHD, only depression, and neither ADHD nor depression. Data from the Tracking Adolescents' Individual Lives Survey (TRAILS) cohort was used in this study. Neuropsychological functioning was assessed in 1549 adolescents, at baseline and follow-up (mean ages 11 and 19 years). The Composite International Diagnostic Interview was used to classify adolescents into 4 groups: ADHD with onset of depression, only ADHD, only depression, and neither ADHD nor depression. Linear mixed effects models were used to analyse group differences in cognitive functioning at baseline and follow-up, and the change in cognitive functioning between these 2 time-points. Results showed a significant main effect of group on response time variability at baseline, working memory maintenance at follow up, and change in response time variability scores between baseline and follow-up. As compared to the healthy and depressed-only groups, adolescents with only ADHD showed longer response time variability at baseline and, which declined between baseline and follow-up. Adolescents with ADHD plus
\end{abstract}

Electronic supplementary material The online version of this article (doi:10.1007/s10802-016-0160-x) contains supplementary material, which is available to authorized users.

Arunima Roy

arunima.roy@mail.mcgill.ca

1 Interdisciplinary Centre Psychopathology and Emotion Regulation (ICPE), University of Groningen, University Medical Centre Groningen, CC 72, P.O. Box 30.001, 9700

RB Groningen, The Netherlands

2 Division of Child Psychiatry, McGill University, Montreal Children's Hospital, Montreal, Quebec, Canada depression showed higher reaction time for working memory maintenance than the depressed only and healthy groups at follow-up. In conclusion, adolescents with self-reported ADHD show poorer cognitive functioning than healthy adolescents and those with only depression. Amongst adolescents with ADHD, specific cognitive domains show poor functioning depending on the presence or absence of comorbid depression. While adolescents with only ADHD have lower reaction time variability, those with comorbid depression have poorer working memory maintenance.

Keywords ADHD · Depression · Cognition · Adolescents

Attention Deficit Hyperactivity Disorder or ADHD is a neurodevelopmental disorder of childhood that often persists into adolescence and adulthood (DSM-5 American Psychiatric Association 2013). Apart from symptoms of hyperactivity, impulsivity and inattentiveness, affected individuals show a wide range of cognitive functioning deficits, such as problems in planning, working memory, inhibition and attention (Cortese et al. 2015; Sebastian et al. 2014; Willcutt et al. 2005). Cognitive dysfunction in ADHD is heterogeneous; not only are cognitive deficits absent in many cases of ADHD, affected cognitive domains also vary widely across individuals with ADHD (Martel et al. 2011). There is, thus, poor consensus regarding the specific cognitive profile associated with ADHD (Nigg et al. 2005; Sergeant et al. 2003; Sergeant et al. 2002; Sjowall et al. 2013; Sonuga-Barke 2003). A suspected source of this variability may be the high comorbidity of ADHD with other psychiatric disorders (Pauli-Pott et al. 2014). Comorbidities may increase the severity of existing cognitive deficits in children and adolescents with ADHD (Crawford et al. 2006). In addition, comorbid disorders may modify the cognitive functioning profile associated 
with ADHD. Although not much is known yet about the deficits associated with ADHD in combination with specific comorbid disorders, it is likely that cognitive profiles differ for each of these conditions (Larochette et al. 2011; Pauli-Pott et al. 2014; Vloet et al. 2010; Willcutt et al. 2001). In this study we will focus on the cognitive functioning and development of depression in adolescents with and without ADHD.

Depression develops in 30 to $70 \%$ of individuals with ADHD (Chronis-Tuscano et al. 2010; Fergusson et al. 2010; Jensen et al. 1993; Meinzer et al. 2014) Results from these studies show that both boys and girls with ADHD, across a wide age range from 4 to 18 years, are likely to develop depression. Comorbid depression leads to further impairments in prognosis and quality of life (Angold et al. 1999; Bagwell et al. 2001; Biederman et al. 1993; Blackman et al. 2005; Coleman 2008). A knowledge of the cognitive profile associated with ADHD and comorbid depression may assist in understanding the ADHD-depression relationship better. Not many studies though, exist on the cognitive functioning of individuals with ADHD and depression. A recent metaanalysis indicated that depression is weakly to moderately associated with deficits in various cognitive domains (Snyder 2013), several of which are also common to ADHD (Nigg 2005).

The combined condition of ADHD and depression may give rise to a unique cognitive profile that is distinguishable from that of either disorder alone (either ADHD or depression) in the following manners. First, as found in previous studies, comorbidities may increase the severity of cognitive deficits (Crawford et al. 2006). Therefore, individuals with ADHD plus depression may have a worse cognitive functioning than those with either ADHD or depression. Second, cognitive deficits may be a vulnerability factor, facilitating the onset of depression (Austin et al. 2001). Thus, in adolescents with ADHD too, cognitive dysfunction may precede an onset of depression. Prospective studies on long-term outcomes of cognitive (dys)function in ADHD are limited and consequently this idea has not been explored yet. Third, cognitive maturation is often delayed amongst individuals with ADHD (Rajendran et al. 2013; Shaw et al. 2006, 2007). A development of depression during this period may further interfere with the process of cognitive maturation and thereby differentiate cases of ADHD plus depression from cases with either ADHD or depression.

This study aims to understand if ADHD with an onset of depression is associated with a unique cognitive profile. In a large population-based sample with retrospective self-reports of ADHD and depression, we assess cognitive functioning differences at two time-points among adolescents with ADHD plus depression, only ADHD, and controls with or without depression. Further, we also assess group differences in the change in cognitive functioning between the two-time points.

\section{Methods}

\section{Cohort}

The data were collected as part of the TRacking Adolescents' Individual Lives Survey (TRAILS), an ongoing Dutch prospective cohort study on psychosocial development and mental health of adolescents. TRAILS involves bi- or triennial measurements from ages 11 onwards and consists of two separate cohorts: one population-based and another clinic-based. (de Winter et al. 2005; Huisman et al. 2008; Oldehinkel et al. 2015b; Ormel et al. 2012). This study is based on data from the TRAILS population cohort.

Children were recruited from five municipalities in the north of The Netherlands, including both urban and rural areas. Primary school participation was requisite for inclusion. Of the 2935 children who met these criteria, 2230 (76.0\%) provided informed consent from both parent and child to participate in the study. The present study utilises data from the first and fourth wave. The first wave (T1) ran from March 2001 to July 2002, the fourth (T4) from October 2008 to September 2010 (T4). Mean age at T1 was 11.1 years $(\mathrm{SD}=0.56)$, and $50.8 \%$ were girls. Response rate at $\mathrm{T} 4$ was $83.4 \%(N=1881$, mean age $=19.1, S D=0.60,52.3 \%$ girls $)$, of whom $84.2 \%(N=1584)$ completed the below-described diagnostic interview. (For an overview of the mean age and gender distributions of participants please see supplementary material, S1). To ensure all onsets of depression were between $\mathrm{T} 1$ and T4, we excluded participants who developed a depression prior to T1 $(n=35)$. This gave a final sample size of 1549 adolescents. T1 is referred to as baseline and T4 as follow-up in following sections of the paper.

The Amsterdam Neuropsychological Tasks used in this study to assess cognitive function was also part of two previous TRAILS publications on depressive problems in adolescents, but not specific to ADHD (Nederhof et al. 2014; Oldehinkel et al. 2015a). These studies used different ANT parameters than here focusing on either attention style (Nederhof et al. 2014) or emotion recognition (Oldehinkel et al. 2015a) in relation to depressive symptoms (rather than disorder). Further, data from the TRAILS cohort has been used in two studies to study mediators (other than cognitive function) of the ADHD-depression association (Roy et al. 2015; Roy et al. 2014). Thus, the content of the current manuscript does not overlap with these previous publications.

\section{Measures}

Cognitive functions were assessed at baseline and follow-up using the Amsterdam Neuropsychological Tasks program (ANT) (please see supplementary material S2 for further details). The ANT has proven to be a sensitive and valid tool in assessing cognitive functions (de Sonneville et al. 1993), 
especially for population and clinic based samples of attention-deficit/hyperactivity disorder (Hanisch et al. 2004; SlaatsWillemse et al. 2003). Previously, studies have used the ANT to assess sustained attention, processing speed (Huijbregts et al. 2002), focused attention, working memory, cognitive flexibility (Lazeron et al. 2006) and response inhibition (Groot et al. 2004). A detailed description of the ANT is available in previous studies (Nederhof et al. 2014; van Deurzen et al. 2012). In short, the ANT is a computer-aided test to assess cognitive capacities, with high sensitivity and validity. We used five subtasks from the ANT: (a) Baseline Speed task, (b) Pattern Recognition task, (c) Sustained Attention - dots task, (d) Memory Search - letters task, and (e) Shifting Attentional Set - visual task. All children were tested individually by trained undergraduate psychology students. Use of prescribed medications (if any) was not withheld prior to the assessments. Verbal instructions, emphasizing both speed and accuracy of performance, and practice trials preceded each task. Six output measures were calculated from the above tasks: processing speed (from Baseline Speed), focussed attention (from Pattern Recognition), response time variability (from Sustained Attention Dots), working memory maintenance (from Memory Search - letters), response inhibition (from Shifting Attentional Set - visual), and cognitive flexibility (from Shifting Attentional Set - visual). For all output measures, except response time variability, mean Reaction Time (RT) in milliseconds was defined as the outcome parameter (for response time variability, within-subject variability in RT of the sustained attention - dots task was defined as the outcome). Only RTs to correct trials were used in the analyses. The Baseline Speed task required responses from both the dominant and the non-dominant hand. The outcome parameter for this task was defined as mean RT of responses from both hands. For all outcomes, RTs with an absolute z-score greater than or equal to 4 were defined as missing (Stevens 2009). Correlations between the measures were generally weak (mean $r=0.24$, range $=0.07-0.55$ ), suggesting limited overlap.

Psychiatric disorders were assessed at follow-up by means of the World Health Organization Composite International Diagnostic Interview (CIDI), version 3.0. The CIDI is a structured diagnostic interview that yields lifetime and current diagnoses according to the definitions and criteria of the Diagnostic and Statistical Manual of Mental Disorders (DSM-IV). The CIDI has been used in a large number of surveys worldwide, and shown to have good concordance with clinical diagnoses (Haro et al. 2006; Kessler et al. 2004, 2009). In addition to the occurrence of psychiatric disorders, the CIDI yields their age at onset and age at last occurrence. The CIDI has good reliability and validity for most diagnoses (Wittchen 1994). In this study, information on CIDI diagnoses of ADHD and depression were used. The ADHD screening questions were operationalised as: (1) a history of concentration problems (such as quickly losing interest in work and games, inability to concentrate on and finish work, not listening to other people when spoken to) prior to the age of 7 that lasted a minimum of 6 months and seemed excessive compared to peers, and/or (2) a history of hyperactivityimpulsivity (such as fidgeting, restlessness and impatience) present before the age of 7 that lasted a minimum of 6 months. A positive response to either of these two questions was followed up by a full DSM-IV based assessment of ADHD. This includes the presence of the requisite number of ADHD symptoms as defined in DSM-IV, an onset before the age of 7 , and the presence of functioning impairments. Depression was operationalized as a lifetime diagnosis of Major Depressive Episode (with or without (hypo)manic symptoms), Dysthymia, or Minor Depressive Disorder. Validity of the CIDI data was supported by prospective parent-, self-, and teacher-reports as assessed with the Child Behaviour Checklist (CBCL), Youth Self Report (YSR), Adult Self Report (ASR), and Teacher's Checklist of Pathology (TCP) from the first wave onwards (supplementary material S3, Tables III-IV) (Achenbach 1991a, b). The TCP, developed by the TRAILS team (de Winter et al. 2005), is based on items from the Teacher's Report Form (Achenbach and Rescorla 2001). As several participants in the TRAILS cohort were from the same class, the TCP was developed to ease the filling up of questionnaires by teachers. Teacher-reported ADHD scores in this study combined the attention and hyperactivity/impulsivity problem items of the TCP (corresponding to the CBCL-YSR attention problems scales).

Information on medication use between baseline and follow-up was collected using parent and self-report questionnaires. Use of anti-depressants included imipramine, clomipramine, amitriptyline, nortriptyline, fluoxetine, citalopram, paroxetine, sertraline, fluvoxamine, escitalopram, moclobemide, venlafaxine; while medications for ADHD involved methylphenidate, atomoxetine, and dexamphetamine.

\section{Analyses}

Based on lifetime CIDI diagnoses at follow-up, participants were categorized into four groups: (1) ADHD with an onset of depression (Group A+D), (2) only ADHD (Group A), (3) only an onset of depression (Group D), and (4) comparison: neither ADHD nor an onset of depression (Group C). Mean selfreported ADHD and depressive symptom scores (assessed using the YSR at T1, T2, T3 and the ASR at T4) at the four assessment waves were plotted to visualise the approximate time-point of divergence in symptom scores amongst the four groups.

Linear mixed effects models were used to analyse differences in cognitive functioning among these groups at baseline and follow-up as well as change in cognitive functioning between baseline and follow-up (slope). We adjusted for the 
effects of age at baseline, gender, and medication use in the analyses by including these variables as covariates. The (co)variance matrix was set to unstructured (i.e., freely estimated) for all analyses. For the cognitive outcome measures that showed group differences, we additionally plotted the standardized RTs per group at baseline and follow-up and estimated the effect sizes of comparisons with group $\mathrm{C}$ using Cohen's $d$.

A sensitivity analysis was conducted as a second step to determine if (lack of) differences in cognitive functioning among groups were due to the presence of psychiatric disorders other than ADHD or depression in the comparison group. For this, a new comparison group (Group H: healthy) was formed by excluding participants with CIDI diagnoses of other psychiatric disorders from group $\mathrm{C}$, and the abovedescribed linear mixed effects analyses were re-run to test cognitive functioning differences among groups $\mathrm{A}+\mathrm{D}, \mathrm{A}, \mathrm{D}$ and $\mathrm{H}$. Age at baseline, gender and medications were included as covariates.

A second sensitivity analysis was additionally performed to explore the validity of the CIDI diagnoses. Data on parentreported ADHD as assessed by the Diagnostic Interview Schedule for Children (DISC) were available for the parallel TRAILS Clinical (high-risk) Cohort at baseline. A comparison of the DISC with the CIDI outcomes showed that almost $80 \%$ of children with an ADHD diagnosis at baseline according to the DISC did not have a CIDI diagnosis of ADHD in adulthood (note that both interviews required an onset of ADHD before age 7). Conversely, $80 \%$ of those who had a CIDI diagnosis of ADHD also had a DISC diagnosis of ADHD in childhood. These results suggest the presence of relatively few false positives in our current sample (with the use of CIDI), yet many false negatives. To assess the possibility of false negative ADHD diagnoses using the CIDI, we reformed the comparison group by excluding participants with a high baseline CBCL or TCP ADHD symptom score (i.e., scores higher than $1.5 S D$ in the total sample).

Analyses were performed using SPSS v. 22.0.0 (IBM Corp., Armonk, NY) and graphs were plotted using MATLAB 2012b (The MathWorks, Inc. Natick, MA). All tests were two-tailed and a $p \leq 0.05$ was considered statistically significant. For the post-hoc pairwise tests, results were adjusted using the Benjamini-Hochberg False Discovery Rate (FDR) (Benjamini and Hochberg 1995) and the threshold for statistical significance was set at $p \leq 0.05$.

\section{Results}

Of the 1549 adolescents in our study, and based on the CIDI, $3.6 \%$ received a diagnosis of ADHD $(n=56)$, and $19.6 \%$ a diagnosis of depression $(n=303)$. Amongst those with ADHD, $37.5 \%$ developed depression between baseline and follow-up $(n=21)$. These numbers are consistent with estimates of lifetime mental health problems obtained in late adolescence (Merikangas et al. 2010). Of the total sample, $37.5 \%$ of adolescents with ADHD and $5.6 \%$ of adolescents with depression received medication at some point between the four assessment waves. Participants with and without medications did not differ in their cognitive functioning scores at baseline or follow-up (for further details please see supplementary material S3 [Tables I-II]).

Parent- (CBCL) and teacher- (TCP) reports, available at baseline, were used to estimate the number of adolescents in the $\mathrm{A}+\mathrm{D}$ and A groups with ADHD scores $>1.5$ SD. Parentreports showed $>1.5 S D$ scores in 5 participants from group $\mathrm{A}+\mathrm{D}(n=21)$ and 12 participants from group $\mathrm{A}(n=35)$. Using teacher-reports, these numbers were five in group A+ $\mathrm{D}$ and seven in group A. Scores greater than 1.5 SD on either parent or teacher reports were found among 10 participants from group $\mathrm{A}+\mathrm{D}$ and 13 participants from group $\mathrm{A}$. Mean parent-reported ADHD symptom scores in group $\mathrm{A}+\mathrm{D}$ and A were $0.87(S D=0.58)$ and $1.01(S D=0.45)$, respectively. Mean teacher-reported ADHD symptom scores were 1.41 $(S D=1.20)$ and $1.16(S D=1.18)$ for groups $\mathrm{A}+\mathrm{D}$ and $\mathrm{A}$, respectively.

Figure 1 presents mean self-reported ADHD and depressive symptom scores assessed (using the YSR and ASR) at each time point for the four groups. At T1, group $A+D$ had higher self-reported ADHD symptoms than group $\mathrm{C}(d=0.53$, $p=.019)$, while group A had higher ADHD symptoms than groups $\mathrm{D}(d=0.67, p<.001)$ and $\mathrm{C}(d=0.88, p<.001)$. ADHD symptom scores at T4 of group $\mathrm{A}+\mathrm{D}$ was higher than groups $\mathrm{A}(d=0.66, p=0.004), \mathrm{D}(d=1.41, p<.001)$ and $\mathrm{C}$ $(d=2.05, p<.001)$, and of group A was higher than groups $\mathrm{D}(d=0.66, p<.001)$ and $\mathrm{C}(d=1.24, p<.001)$. Depressive symptoms at $\mathrm{T} 1$ were higher amongst groups $\mathrm{A}+\mathrm{D}(d=0.53$, $p=.023), \mathrm{A}(d=0.48, p=.002)$ and $\mathrm{D}(d=0.43, p<.001)$ than group C. At T4, mean depressive scores were high for group $\mathrm{A}+\mathrm{D}$ as compared to group $\mathrm{A}(d=0.98, p<.001)$, group $\mathrm{D}$ $(d=0.64, p<.001)$ and group $\mathrm{C}(d=1.73, p<.001)$. For group $\mathrm{D}$ at $\mathrm{T} 4$, depressive symptoms were higher than group $\mathrm{A}$ $(d=0.35, p=.010)$ and group $\mathrm{C}(d=1.01, p<.001)$.

Table 1 presents mean age at onsets of ADHD and depression, and mean RTs for cognitive outcome measures in the five groups A+D, A, D, C, and H. Pearson's correlations revealed statistically significant relationships between RTs at baseline and follow-up for all cognitive outcome measures (processing speed: $r=0.44$; focussed attention: $r=0.37$; response time variability: $r=0.50$; working memory maintenance: $r=0.46$; inhibition: $r=0.34$; cognitive flexibility: $r=0.34$; all $p<.001$ ). For further details on the association between baseline and follow-up cognitive functions in the TRAILS sample, please see Boelema et al. (2013).

Linear mixed model analyses with sex, age, and ADHD and depression medication use as covariates revealed significant 

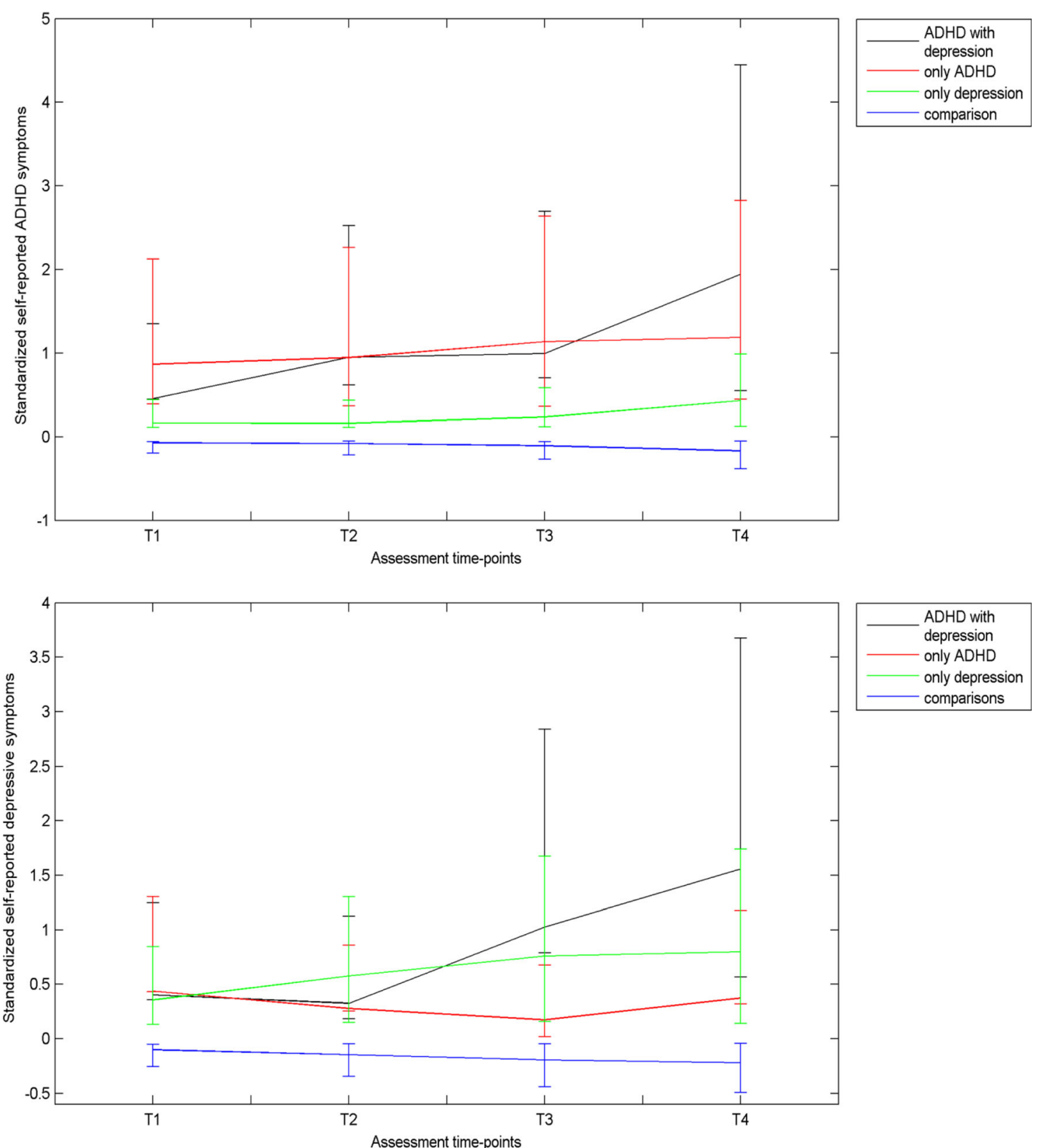

Fig. 1 Mean ADHD and depressive symptom scores (assessed using Youth Self Reports at T1, T2, T3 and Adult Self Reports at T4) between ages 11 and 19 years for all four groups

group differences in response time variability at baseline: $F(3$, $1517)=3.92, p=.008, \eta^{2}=0.004$; working memory maintenance at follow up: $F(3,1515)=2.56, p=.05, \eta^{2}=0.004$, and; change in response time variability scores between baseline and follow-up: $F(3,1515)=3.35, p=.01, \eta^{2}=0.005$. Baseline response time variability RT was higher in group A than group $\mathrm{C}(p=.001)$. Working memory maintenance $\mathrm{RT}$ at follow-up was higher in group $\mathrm{A}+\mathrm{D}$ than group $\mathrm{C}(p=.007)$. Between baseline and follow-up, the slope of change in response time variability RT for group A was significantly more negative $(p=.002)$ than that of group C. The covariates-corrected effect sizes for these three differences were $d=0.44, d=0.60$, and $d=-0.47$, respectively. Finally, results of post-hoc pairwise comparisons are presented in Table 2.

Figure 2 presents RTs of the four groups for response time variability and working memory maintenance functions at the 2 time-points. Linear mixed model analyses on these RT scores (without including covariates) yielded similar group differences in response time variability at baseline: $F(3$, $1521)=4.23, p=.005, \mathrm{n}^{2}=0.003$; working memory maintenance at follow up: $F(3,1521)=2.56, p=.05, \eta^{2}=0.002$, and; change in response time variability scores between baseline and follow-up: $F(3,1516)=3.36, p=.01, \eta^{2}=0.004$ and likewise indicated a higher baseline response time variability 
Table 1 Summary of group characteristics, including age at onset of ADHD and depression, and reaction time for cognitive measures at baseline and follow-up

\begin{tabular}{|c|c|c|c|c|c|}
\hline & \multicolumn{5}{|l|}{ Groups ${ }^{*}$} \\
\hline & $\begin{array}{l}\mathrm{A}+\mathrm{D} \\
N=21, \\
52 \% \text { girls } \\
(\text { mean } \pm \mathrm{SD})\end{array}$ & $\begin{array}{l}\mathrm{A} \\
N=35, \\
37 \% \text { girls } \\
(\text { mean } \pm \mathrm{SD})\end{array}$ & $\begin{array}{l}\mathrm{D} \\
N=282, \\
73 \% \text { girls } \\
(\text { mean } \pm \mathrm{SD})\end{array}$ & $\begin{array}{l}\mathrm{C} \\
N=1211,50 \% \text { girls } \\
(\text { mean } \pm \mathrm{SD})\end{array}$ & $\begin{array}{l}\mathrm{H} \\
N=888, \\
49 \% \text { girls } \\
(\text { mean } \pm \mathrm{SD})\end{array}$ \\
\hline Age at onset of ADHD ${ }^{* *}$ & $6 \pm 2$ & $5 \pm 2$ & - & - & - \\
\hline Age at onset of depression ${ }^{* *}$ & $14 \pm 2.4$ & - & $15 \pm 2.2$ & - & - \\
\hline \multicolumn{6}{|l|}{ Processing speed ${ }^{\#}$} \\
\hline Baseline & $328 \pm 38$ & $345 \pm 42$ & $329 \pm 38$ & $327 \pm 39$ & $325 \pm 37$ \\
\hline Follow-up & $243 \pm 24$ & $256 \pm 25$ & $252 \pm 23$ & $250 \pm 25$ & $250 \pm 24$ \\
\hline \multicolumn{6}{|l|}{ Focused attention $^{\#}$} \\
\hline Baseline & $1467 \pm 604$ & $1533 \pm 356$ & $1462 \pm 469$ & $1420 \pm 443$ & $1404 \pm 442$ \\
\hline Follow-up & $795 \pm 301$ & $787 \pm 238$ & $810 \pm 259$ & $808 \pm 258$ & $799 \pm 252$ \\
\hline \multicolumn{6}{|l|}{ Response time variability ${ }^{\#}$} \\
\hline Baseline & $1898 \pm 956$ & $2095 \pm 762$ & $1688 \pm 828$ & $1652 \pm 810$ & $1617 \pm 806$ \\
\hline Follow-up & $974 \pm 391$ & $916 \pm 365$ & $862 \pm 366$ & $858 \pm 366$ & $849 \pm 367$ \\
\hline \multicolumn{6}{|c|}{ Working memory maintenance ${ }^{\#}$} \\
\hline Baseline & $621 \pm 331$ & $576 \pm 244$ & $499 \pm 267$ & $517 \pm 265$ & $517 \pm 267$ \\
\hline Follow-up & $347 \pm 173$ & $276 \pm 174$ & $257 \pm 149$ & $255 \pm 146$ & $252 \pm 145$ \\
\hline \multicolumn{6}{|l|}{ Cognitive flexibility ${ }^{\#}$} \\
\hline Baseline & $637 \pm 241$ & $645 \pm 210$ & $644 \pm 250$ & $622 \pm 236$ & $621 \pm 236$ \\
\hline Follow-up & $374 \pm 143$ & $356 \pm 126$ & $377 \pm 161$ & $348 \pm 146$ & $341 \pm 133$ \\
\hline \multicolumn{6}{|l|}{ Response inhibition ${ }^{\#}$} \\
\hline Baseline & $214 \pm 173$ & $235 \pm 190$ & $251 \pm 191$ & $248 \pm 183$ & $245 \pm 185$ \\
\hline Follow-up & $245 \pm 207$ & $179 \pm 189$ & $210 \pm 171$ & $201 \pm 164$ & $202 \pm 163$ \\
\hline
\end{tabular}

*A+D ADHD with onset of depression, $A$ only ADHD, $D$ only depression, $C$ comparison; neither ADHD nor depression, $H$ healthy; no psychiatric diagnoses

**in years; \#Reaction time in milliseconds

RT in group A than group C $(p=.001)$; higher working memory maintenance $\mathrm{RT}$ at follow-up in group $\mathrm{A}+\mathrm{D}$ than group $\mathrm{C}$ $(p=.007)$; and a more negative slope of change in response time variability RT for group A relative to group C ( $p=.002)$. The effect sizes for these three differences were $d=0.52$, $d=0.60$, and $d=-0.55$, respectively.
For the first sensitivity analysis, participants from group $\mathrm{C}$ with psychiatric diagnoses (other than ADHD or depression) were excluded (separation anxiety: $n=19$; agoraphobia: $n=6$; conduct disorder: $n=71$; generalised anxiety disorder: $n=22$; oppositional defiant disorder: $n=65$; panic disorder: $n=13$; separation anxiety disorder: $n=25$; social phobia: $n=110$;
Table 2 Posthoc pairwise comparisons of groups for differences in reactions times for response time variability and working memory maintenance

\begin{tabular}{|c|c|c|c|c|c|c|}
\hline \multirow[t]{2}{*}{ Groups compared ${ }^{*}$} & \multicolumn{3}{|c|}{ Response time variability-baseline } & \multicolumn{3}{|c|}{ Working memory maintenance-follow-up } \\
\hline & Mean difference & SE & $\mathrm{p}^{\#}$ & Mean difference & $\mathrm{SE}$ & $\mathrm{p}^{\#}$ \\
\hline $\mathrm{A}+\mathrm{D} \mathrm{v} / \mathrm{s} \mathrm{A}$ & -123.74 & 147.66 & 0.40 & 64.38 & 50.19 & 0.20 \\
\hline $\mathrm{A}+\mathrm{D} \mathrm{v} / \mathrm{s} \mathrm{D}$ & 134.99 & 121.22 & 0.26 & 97.79 & 41.28 & 0.01 \\
\hline $\mathrm{A}+\mathrm{D} v / \mathrm{s} \mathrm{C}$ & 151.50 & 117.95 & 0.19 & 95.97 & 40.16 & 0.01 \\
\hline $\mathrm{A} v / \mathrm{s} \mathrm{D}$ & 258.74 & 96.71 & 0.008 & 33.40 & 32.94 & 0.31 \\
\hline $\mathrm{A} v / \mathrm{s} \mathrm{C}$ & 275.25 & 92.45 & 0.003 & 31.59 & 31.47 & 0.31 \\
\hline $\mathrm{D}$ v/s C & 16.51 & 35.78 & 0.64 & -1.81 & 12.22 & 0.88 \\
\hline
\end{tabular}

* Groups: $A+D$ ADHD with depression, $A$ only ADHD, $D$ only depression, $C$ comparison

\# $p$ values adjusted for multiple testing using the Benjamini-Hochberg False Discovery Rate 
Fig. 2 Reaction times for working memory maintenance and response time variability at baseline and follow-up
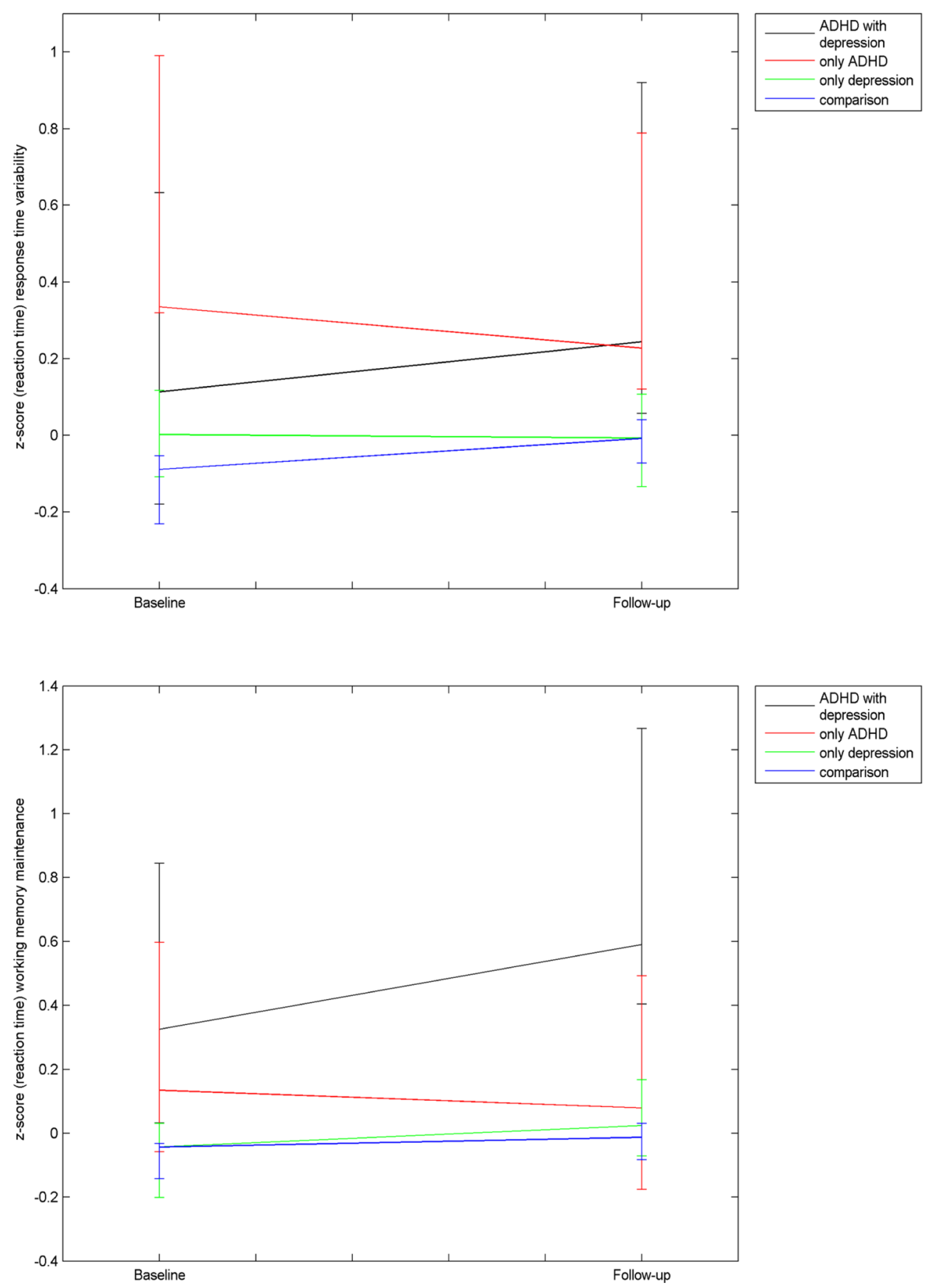

specific phobia: $n=110$, total $n=323$ ) to form a new group of 888 adolescents (group H; healthy). Linear mixed effects analyses revealed group differences in baseline response time variability: $F(3,1207)=4.64, p=.003, \eta^{2}=0.007$; change in response time variability between baseline and follow-up: $F(3$, 1200) $=3.99, p=.008, \eta^{2}=0.005$, and; follow-up working memory maintenance: $F(3,1198)=2.76, p=.041, \eta^{2}=0.005$. Results of group and posthoc pairwise comparisons for response time variability and working memory maintenance were comparable to results from the main analysis. In addition, group differences in baseline processing speed were found: $F(3,1201)=2.90, p=.03, \eta^{2}=0.006$. None of the group differences in processing speed remained significant after applying FDR corrections.

For the second sensitivity analysis, 158 participants with a CBCL or TCP ADHD score with $S D>1.5$ were excluded from the comparison group to form a new comparison group of 1391 adolescents. Linear mixed effects analyses revealed group differences in baseline response time variability: $F(3$, $1373)=7.05, p<.001, \eta^{2}=0.007$; follow-up working memory 
maintenance: $F(3,1358)=3.62, p=.013, \eta^{2}=0.005$, and; change in response time variability between baseline and follow-up: $F(3,1369)=5.42, p=.001, \eta^{2}=0.006$. Results of group and posthoc pairwise comparisons for response time variability and working memory maintenance were comparable to the results from the main analysis. Additionally, group differences were found in baseline processing speed: $F(3$, $1353)=3.13, p=.025, \eta^{2}=0.006$ ). Post hoc pairwise comparisons showed that group A was significantly slower (FDR corrected) than group $\mathrm{C}$ (mean difference $=12.78, S E=4.63$, $p=.006$ ).

\section{Discussion}

This study was aimed at assessing if ADHD with an onset of depression is associated with a unique cognitive functioning profile in adolescents with retrospectively self-reported ADHD and depression. Results show that cognitive functioning in adolescents with ADHD plus depression and only ADHD differed from the control groups either with or without depression. In particular, working memory maintenance of adolescents with ADHD plus depression at mean age 19 years was poor as compared to healthy adolescents and adolescents with only depression. Furthermore, the group of adolescents with only ADHD performed worse in response time variability at mean age 11 than the depressed and comparison groups. Our results also suggest that response time variability function improved between early adolescence and young adulthood in adolescents with only ADHD. We found no evidence, however, for cognitive functioning differences between adolescents with ADHD who did and did not develop depression.

The presence of comorbidities in individuals with ADHD is believed to increase the severity of cognitive deficits (Crawford et al. 2006). Consequently, we suspected that adolescents with ADHD and comorbid depression may have a poorer cognitive functioning than adolescents with only ADHD. We found no differences specifically between these two groups on our measures. Further, based on previous research, we suspected that an onset of depression in adolescents with ADHD may be preceded by impaired cognitive functioning (Austin et al. 2001). Our results did not support this either; adolescents with ADHD who developed depression did not differ from healthy comparisons at the baseline assessments. We also suspected that the development of depression would interfere with the process of cognitive maturation in adolescents with ADHD. Results show that adolescents with only ADHD showed an improvement in their cognitive (response time variability) functioning between ages of 11 and 19 years. Adolescents with ADHD who developed additional depression, did not show any cognitive improvements.
Adolescents with ADHD plus an onset of depression showed poorer working memory maintenance than comparison adolescents at a mean age of 19 years. The presence of memory maintenance difficulties at late adolescence in our sample may be related to the development of comorbid depression in adolescents with ADHD. Based on self-reported symptoms, we found that these adolescents showed increasing depressive symptoms for at least 6 years between the second and fourth assessment waves. ADHD symptom scores, on the other hand, did not change much for this comorbid group. It is possible that the upcoming depression and not ADHD played a role in the poor working memory maintenance performance at late adolescence. However, adolescents with only depression had a similarly increasing depressive symptom profile but did not show poor cognitive functioning in any domain (possible reasons for which we discuss later). It can be speculated that the development of depression may have increased the cognitive burden associated with ADHD and led to working memory maintenance difficulties. Alternately, poor cognitive functioning may have led to the development of depression in adolescents with ADHD.

Working memory maintenance, at age 11 , of adolescents with ADHD and either with or without depression was comparable to that of the comparison group. Previous studies though, show impaired working memory in children with ADHD at an early age. Three related reasons as to why we did not find any group differences in working memory maintenance at age 11 in this study are worth mentioning. First, previous studies have been mostly based on recall tasks, which unlike recognition based tasks, such as the memory search task of the ANT, are more likely to show differences between children with and without ADHD early on (Rapport et al. 2000). Second, working memory maintenance assessed by visual array tasks, as in the ANT, is the total information held in working memory at any given point (Rapport et al. 2013; Shipstead et al. 2012). This component of working memory has been shown to be only minimally affected in children with ADHD (Raiker et al. 2012). In contrast, the central executive components of working memory, as assessed by complex span tasks, differ from working memory maintenance by being involved in the active processing of information held internally and are not related to the storage or maintenance of information. The central executive components of working memory are more often impaired than working memory maintenance in children with ADHD (Kasper et al. 2012; Rapport et al. 2013; Shipstead et al. 2012). Third, it is possible that the memory search task of the ANT measures short-term memory (Cowan 2008), which is likewise less impaired in children with ADHD (Dovis et al. 2015).

Adolescents with only ADHD showed poorer response time variability than comparison adolescents. Response time variability performance, however, improved with time in the group with only ADHD. For this group, self-reported ADHD 
symptoms also showed a decrease between early and late adolescence. It is likely that a decline in ADHD symptom severity was related to the improving response time variability performance.

Amongst adolescents with ADHD, results showed that the response time variability domain was affected at early adolescence while working memory maintenance was affected at young adulthood. The maturation of various cognitive domains occurs at different ages (Anderson 2002; Anderson et al. 2001; Boelema et al. 2013; Huizinga et al. 2006; Luna and Sweeney 2004), which may explain why response time variability was affected first followed by working memory maintenance later and not the other way around. The maturation of working memory peaks late in adolescence (Boelema et al. 2013; Huizinga et al. 2006; Luna and Sweeney 2004) and response time variability may therefore be affected prior to working memory maintenance.

Previous studies have reported cognitive flexibility, focused attention, and response inhibition impairments in individuals with ADHD (Nigg 2005). We did not find deficits in these three cognitive domains amongst adolescents with ADHD. One explanation for this could relate to the age of assessments in our sample (van Lieshout et al. 2013). The vast majority of research on ADHD-related cognitive deficits involves younger children, while we included adolescent participants. Further, core cognitive deficits proposed to be etiologically related to ADHD are less evidently present in adolescence (despite the presence of ADHD), suggesting that some children have sufficient cognitive maturation and do not show deficits (Thissen et al. 2014). Moreover, various cognitive domains mature at different ages (Anderson 2002; Anderson et al. 2001; Boelema et al. 2013; Luna and Sweeney 2004). It is thus, possible that several cognitive functions had matured sufficiently in our sample by the first assessment time point and therefore did not show group differences.

Some studies have suggested that only response inhibition deficits persist in the longer term while other cognitive domains mature sufficiently irrespective of remission from ADHD (Lei et al. 2015; McAuley et al. 2014). In this respect, our results may be remarkable in showing a lack of response inhibition deficits at young adulthood. However, these studies were based on patient populations of ADHD with severe problems and often multiple comorbidities. Our research findings are based on ADHD cases selected from the general population, who may not show severe cognitive functioning problems. Another alternative explanation comes from recent studies suggesting that ADHD-related cognitive deficits may be seen only for domains of working memory and sustained attention (Rapport et al. 2013), but not response inhibition (Alderson et al. 2007; Lijffijt et al. 2005). A final explanation for not finding response inhibition deficits, as in many previous studies that reported no deficits, may be related to the task differences among studies. Withholding a response on some trials (such as in the Stop task and Go/NoGo task) and withholding a compatible and automatic response on all trials (as in the ANT) may tap into different cognitive processes (Rommelse et al. 2007). Overall, the association of response inhibition deficits with ADHD is widely debated and further research may be needed to fully understand the heterogeneity in current literature.

A recent review of the literature has revealed that several domains of cognitive functioning including flexibility, attention, speed, and inhibition are affected in individuals with depression (Snyder 2013). Our results did not show impaired functioning in any domain for adolescents with only depression. The majority of studies on cognitive dysfunction in depressed individuals are limited to adulthood or even lateadulthood (Han et al. 2012). Cognitive dysfunction in adolescents with depression may not necessarily be similar to that in adults. It is also likely that depression-related cognitive deficits are not present pre-morbidly or shortly after onset, but develop only gradually if the depression persists or re-occurs over time. In this case, these deficits may not have fully emerged in our sample of young adults with relatively recently developed depressive problems.

An important limitation of our study concerns the use of the CIDI to establish diagnoses of ADHD. Based on parent and teacher reports available at baseline, it was found that ADHD symptom scores were low in the CIDI diagnosed ADHD groups: less than half the participants in the two ADHD groups scored greater than 1.5 standard deviations on either the parent or teacher ratings. The CIDI is a well-validated interview (Wittchen 1994), but the ADHD section is of a relatively recent date, and has therefore been used in only a limited number of studies so far (de Graaf et al. 2008; Kessler et al. 2006, 2010; Lara et al. 2009). The CIDI assesses the presence of self-reported symptoms during a structured interview which are used to construct lifetime as well as current diagnoses according to the DSM-IV. The CIDI- based ADHD diagnosis includes the presence of functioning impairments. Given the age of the sample at the time of the diagnostic interview (about 19 years) and the availability of fully standardized diagnostic interviews at this age, we used the best possible interview to determine the presence or absence of ADHD. Furthermore, the prevalence rates derived from the CIDI are comparable to that obtained from other populationbased studies in adulthood (Merikangas et al. 2010). Although previous studies have used information form the respondent to establish ADHD diagnoses in adulthood (de Graaf et al. 2008; Kessler et al. 2006; Lara et al. 2009), in childhood this practice is uncommon. The use of the respondent-only assessments as opposed to multiple-informant based assessments, may underestimate the actual prevalence of ADHD or misdiagnose individuals as having ADHD (Sibley et al. 2012), and this likely also holds for adults (Privitera et al. 2015). Young adults, like children and adolescents, may not have a full appreciation of 
their symptoms or impairments and in our sample we may have found additional individuals with a diagnosis of ADHD had we also conducted a parent-interview. Currently, those individuals with (possible) parent but not selfrecognized ADHD are part of the no problem group and this misclassification may have led to finding less outspoken cognitive differences between the groups. However, additional post-hoc analyses indicated that removing participants with a high parent/teacher ADHD score at baseline and without a CIDI ADHD diagnosis yielded highly similar findings. One exception was that children with ADHD only were slower on baseline speed at age 11 . We conclude that current findings pertain to participants diagnosed with ADHD who themselves recognize their childhood onset ADHD symptoms and impairments. We have nonetheless no reason to doubt the validity of these findings since removal of possible false negatives (i.e. cases we would have identified through a parent interview) did not alter the conclusions of our study.

Other limitations to the manuscript include the following: first, the number of adolescents with ADHD only and ADHD combined with depression was low in our sample. Because of this, some group differences may not have reached statistical significance. Conversely, some group differences may have reached significance at smaller effect sizes. Second, as the incidence of depression remains high after 19 years of age, a part of the group with only ADHD (as well as part of the control group) may still develop depression later on. This may have led to an incorrect grouping of adolescents, and possible under-estimation of effects. Third, we did not withdraw participants from stimulant medication prior to cognitive testing although this did not seem to influence our findings.

Thus far, it has been difficult to pinpoint the exact cognitive profile associated with ADHD with affected cognitive domains varying widely across studies. Cognitive function is assumed to change throughout development in patients with ADHD and to generally improve with time (Coghill et al. 2014; Halperin and Schulz 2006; Rajendran et al. 2013). These age-dependent changes may explain some of the discrepancies found in studies. Apart from age-related cognitive deficits, the presence of comorbidities may also explain the heterogeneity of cognitive profiles associated with ADHD. Previously, studies have shown that comorbid anxiety and oppositional defiant disorder can markedly change the cognitive profiles of children and adolescents with ADHD (Rhodes et al. 2012; Vance et al. 2013). Our results also show cognitive differences amongst adolescents with and without comorbid depression, and further reveal that these profiles vary with age. Future studies may benefit from including assessments of comorbidities and age-dependent changes to better capture the nature of cognitive functioning associated with ADHD.

Acknowledgments This research is part of the TRacking Adolescents' Individual Lives Survey (TRAILS). Participating centers of TRAILS include various departments of the University Medical Center and University of Groningen, the Erasmus University Medical Center Rotterdam, the University of Utrecht, the Radboud Medical Center Nijmegen, and the Parnassia Bavo group, all in the Netherlands. TRAILS has been financially supported by various grants from the Netherlands Organization for Scientific Research NWO (Medical Research Council program grant GB-MW 940-38-011; ZonMW Brainpower grant 100-001-004; ZonMw Risk Behavior and Dependence grants 60-60600-98-018 and 60-60600-97-118; ZonMw Culture and Health grant 261-98-710; Social Sciences Council mediumsized investment grants GB-MaGW 480-01-006 and GB-MaGW 480-07-001; Social Sciences Council project grants GB-MaGW 457-03-018, GB-MaGW 452-04-314, and GB-MaGW 452-06-004; NWO large-sized investment grant 175.010.2003.005; NWO Longitudinal Survey and Panel Funding 481-08-013); the Sophia Foundation for Medical Research (projects 301 and 393), the Dutch Ministry of Justice (WODC), the European Science Foundation (EuroSTRESS project FP-006), Biobanking and Biomolecular Resources Research Infrastructure BBMRI-NL (CP 32), the participating universities, and the Accare Centre for Child and Adolescent Psychiatry. We are grateful to all participating adolescents and parents and to everyone who worked on this project and made it possible.

\section{Compliance with Ethical Standards}

Conflict of Interest The authors report no biomedical financial interests or potential conflicts of interests.

Ethical Approval The study was approved by the Dutch Central Committee on Research InvolvingHuman Subjects (CCMO). All procedures were performed in accordance with the ethical standards of the institutional research committee and with the 1964 Helsinki declaration and its later amendments or comparable ethical standards.

Informed Consent Informed consent was obtained from all individual participants included in the study and all measurements were carried out with their adequate understanding and written consent.

Open Access This article is distributed under the terms of the Creative Commons Attribution 4.0 International License (http:// creativecommons.org/licenses/by/4.0/), which permits unrestricted use, distribution, and reproduction in any medium, provided you give appropriate credit to the original author(s) and the source, provide a link to the Creative Commons license, and indicate if changes were made.

\section{References}

Achenbach, T. M. (1991a). Manual for the child behavior checklist/4-18 and 1991 profile. Burlington: University of Vermont.

Achenbach, T. M. (1991b). Manual for the youth self-report and 1991 profile. Burlington: University of Vermont.

Achenbach, T. M., \& Rescorla, L. A. (2001). Manual for the ASEBA school-age forms and profiles. Burlington: University of Vermont.

Alderson, R. M., Rapport, M. D., \& Kofler, M. J. (2007). Attentiondeficit/hyperactivity disorder and behavioral inhibition: a metaanalytic review of the stop-signal paradigm. Journal of Abnormal Child Psychology, 35, 745-758. doi:10.1007/s10802-007-9131-6.

Anderson, P. (2002). Assessment and development of executive function (EF) during childhood. Child Neuropsychology, 8, 71-82.

Anderson, V. A., Anderson, P., Northam, E., Jacobs, R., \& Catroppa, C. (2001). Development of executive functions through late childhood 
and adolescence in an Australian sample. Developmental Neuropsychology, 20, 385-406.

Angold, A., Costello, E. J., \& Erkanli, A. (1999). Comorbidity. Journal of Child Psychology and Psychiatry, and Allied Disciplines, 40, 57-87.

Austin, M. P., Mitchell, P., \& Goodwin, G. M. (2001). Cognitive deficits in depression: possible implications for functional neuropathology. The British Journal of Psychiatry: the Journal of Mental Science, 178, 200-206.

Bagwell, C. L., Molina, B. S., Pelham, W. E., Jr., \& Hoza, B. (2001). Attention-deficit hyperactivity disorder and problems in peer relations: predictions from childhood to adolescence. Journal of the American Academy of Child and Adolescent Psychiatry, 40, 12851292. doi:10.1097/00004583-200111000-00008.

Benjamini, Y., \& Hochberg, Y. (1995). Controlling the false discovery rate: a practical and powerful approach to multiple testing. Journal of the Royal Statistical Society Series B (Methodological), 289-300.

Biederman, J., Faraone, S. V., Spencer, T., Wilens, T., Norman, D., Lapey, K. A., . . \& Doyle, A. (1993). Patterns of psychiatric comorbidity, cognition, and psychosocial functioning in adults with attention deficit hyperactivity disorder. The American Journal of Psychiatry, 150, 1792-1798.

Blackman, G. L., Ostrander, R., \& Herman, K. C. (2005). Children with ADHD and depression: a multisource, multimethod assessment of clinical, social, and academic functioning. Journal of Attention Disorders, 8, 195-207. doi:10.1177/1087054705278777.

Boelema, S. R., Harakeh, Z., Ormel, J., Hartman, C. A., Vollebergh, W. A., \& van Zandvoort, M. J. (2013). Executive functioning shows differential maturation from early to late adolescence: Longitudinal findings from a TRAILS study.

Chronis-Tuscano, A., Molina, B. S., Pelham, W. E., Applegate, B., Dahlke, A., Overmyer, M., \& Lahey, B. B. (2010). Very early predictors of adolescent depression and suicide attempts in children with attention-deficit/hyperactivity disorder. Archives of General Psychiatry, 67, 1044-1051. doi:10.1001/archgenpsychiatry.2010. 127.

Coghill, D. R., Hayward, D., Rhodes, S. M., Grimmer, C., \& Matthews, K. (2014). A longitudinal examination of neuropsychological and clinical functioning in boys with attention deficit hyperactivity disorder (ADHD): improvements in executive functioning do not explain clinical improvement. Psychological Medicine, 44, 10871099. doi:10.1017/S0033291713001761.

Coleman, W. L. (2008). Social competence and friendship formation in adolescents with attention-deficit/hyperactivity disorder. Adolescent Medicine: State of the Art Reviews, 19, 278299. $\mathbf{x}$.

Cortese, S., Ferrin, M., Brandeis, D., Buitelaar, J., Daley, D., Dittmann, R. W., . . \& \& European ADHD Guidelines Group (EAGG) (2015). Cognitive training for attention-deficit/hyperactivity disorder: Meta-analysis of clinical and neuropsychological outcomes from randomized controlled trials. Journal of the American Academy of Child and Adolescent Psychiatry, 54, 164-174. doi:10.1016/j.jaac. 2014.12.010

Cowan, N. (2008). What are the differences between long-term, short-term, and working memory? Progress in Brain Research, 169, 323-338.

Crawford, S. G., Kaplan, B. J., \& Dewey, D. (2006). Effects of coexisting disorders on cognition and behavior in children with ADHD. Journal of Attention Disorders, 10, 192-199.

de Graaf, R., Kessler, R. C., Fayyad, J., ten Have, M., Alonso, J., Angermeyer, M., ... \& Posada-Villa, J. (2008). The prevalence and effects of adult attention-deficit/hyperactivity disorder (ADHD) on the performance of workers: Results from the WHO World Mental Health Survey Initiative. Occupational and Environmental Medicine, 65, 835-842.

de Sonneville, L. M., Geeraets, M. H., \& Woestenburg, J. C. (1993). Information processing in children with minor neurological dysfunction: behavioural and neurophysiological indices. Early Human Development, 34, 69-78.

de Winter, A. F., Oldehinkel, A. J., Veenstra, R., Brunnekreef, J. A., Verhulst, F. C., \& Ormel, J. (2005). Evaluation of non-response bias in mental health determinants and outcomes in a large sample of preadolescents. European Journal of Epidemiology, 20, 173-181.

Dovis, S., Van der Oord, S., Huizenga, H. M., Wiers, R. W., \& Prins, P. J. (2015). Prevalence and diagnostic validity of motivational impairments and deficits in visuospatial short-term memory and working memory in ADHD subtypes. European Child \& Adolescent Psychiatry, 24, 575-590.

DSM-5 American Psychiatric Association. (2013). Diagnostic and statistical manual of mental disorders. Arlington: American Psychiatric Publishing.

Fergusson, D. M., Boden, J. M., \& Horwood, L. J. (2010). Classification of behavior disorders in adolescence: scaling methods, predictive validity, and gender differences. Journal of Abnormal Psychology, 119, 699-712. doi:10.1037/a0018610.

Groot, A. S., de Sonneville, L. M., Stins, J. F., \& Boomsma, D. I. (2004). Familial influences on sustained attention and inhibition in preschoolers. Journal of Child Psychology and Psychiatry, and Allied Disciplines, 45, 306-314.

Halperin, J. M., \& Schulz, K. P. (2006). Revisiting the role of the prefrontal cortex in the pathophysiology of attention-deficit/hyperactivity disorder. Psychological Bulletin, 132, 560-581.

Han, G., Klimes-Dougan, B., Jepsen, S., Ballard, K., Nelson, M., Houri, A., . . \& \& Cullen, K. (2012). Selective neurocognitive impairments in adolescents with major depressive disorder. Journal of Adolescence, 35, 11-20.

Hanisch, C., Konrad, K., Günther, T., \& Herpertz-Dahlmann, B. (2004). Age-dependent neuropsychological deficits and effects of methylphenidate in children with attention-deficit/hyperactivity disorder: a comparison of pre-and grade-school children. Journal of Neural Transmission, 111, 865-881.

Haro, J. M., Arbabzadeh-Bouchez, S., Brugha, T. S., de Girolamo, G., Guyer, M. E., Jin, R., . . . \& Kessler, R. C. (2006). Concordance of the composite international diagnostic interview version 3.0 (CIDI 3.0) with standardized clinical assessments in the WHO world mental health surveys. International Journal of Methods in Psychiatric Research, 15, 167-180.

Huijbregts, S. C., de Sonneville, L. M., Licht, R., van Spronsen, F. J., Verkerk, P. H., \& Sergeant, J. A. (2002). Sustained attention and inhibition of cognitive interference in treated phenylketonuria: associations with concurrent and lifetime phenylalanine concentrations. Neuropsychologia, 40, 7-15.

Huisman, M., Oldehinkel, A. J., de Winter, A., Minderaa, R. B., de Bildt, A., Huizink, A. C., . . \& \& Ormel, J. (2008). Cohort profile: the Dutch 'TRacking adolescents' individual lives survey; TRAILS. International Journal of Epidemiology, 37, 1227-1235. doi:10. 1093/ije/dym273.

Huizinga, M., Dolan, C. V., \& van der Molen, M. W. (2006). Age-related change in executive function: developmental trends and a latent variable analysis. Neuropsychologia, 44, 2017-2036.

Jensen, P. S., Shervette, R. E., 3rd, Xenakis, S. N., \& Richters, J. (1993). Anxiety and depressive disorders in attention deficit disorder with hyperactivity: new findings. The American Journal of Psychiatry, 150, 1203-1209.

Kasper, L. J., Alderson, R. M., \& Hudec, K. L. (2012). Moderators of working memory deficits in children with attention-deficit/hyperactivity disorder (ADHD): a meta-analytic review. Clinical Psychology Review, 32, 605-617.

Kessler, R. C., Berglund, P., Chiu, W. T., Demler, O., Heeringa, S., Hiripi, E., . . \& \& Zheng, H. (2004). The US national comorbidity survey replication (NCS-R): design and field procedures. International Journal of Methods in Psychiatric Research, 13, 69-92. 
Kessler, R. C., Adler, L., Barkley, R., Biederman, J., Conners, C. K., Demler, O., ... \& Zaslavsky, A. M. (2006). The prevalence and correlates of adult ADHD in the United States: results from the National Comorbidity Survey Replication. The American journal of psychiatry, 163, 716-723.

Kessler, R. C., Avenevoli, S., Green, J., Gruber, M. J., Guyer, M., He, Y., . ..\& Zaslavsky, A. M. (2009). National comorbidity survey replication adolescent supplement (NCS-A): III. concordance of DSM-IV/ CIDI diagnoses with clinical reassessments. Journal of the American Academy of Child and Adolescent Psychiatry, 48, 386399. doi:10.1097/CHI.0b013e31819a1cbc.

Kessler, R. C., Green, J. G., Adler, L. A., Barkley, R. A., Chatterji, S., Faraone, S. V., ... \& Van Brunt, D. L. (2010). Structure and diagnosis of adult attention-deficit/hyperactivity disorder: analysis of expanded symptom criteria from the Adult ADHD Clinical Diagnostic Scale. Archives of General Psychiatry, 67, 1168-1178.

Lara, C., Fayyad, J., De Graaf, R., Kessler, R. C., Aguilar-Gaxiola, S., Angermeyer, M., ... \& Sampson, N. (2009). Childhood predictors of adult attention-deficit/hyperactivity disorder: results from the World Health Organization World Mental Health Survey Initiative. Biological Psychiatry, 65, 46-54.

Larochette, A. C., Harrison, A. G., Rosenblum, Y., \& Bowie, C. R. (2011). Additive neurocognitive deficits in adults with attentiondeficit/hyperactivity disorder and depressive symptoms. Archives of Clinical Neuropsychology: The Official Journal of the National Academy of Neuropsychologists, 26, 385-395. doi:10.1093/arclin/ acr033.

Lazeron, R. H., de Sonneville, L. M., Scheltens, P., Polman, C. H., \& Barkhof, F. (2006). Cognitive slowing in multiple sclerosis is strongly associated with brain volume reduction. Multiple Sclerosis (Houndmills, Basingstoke, England), 12, 760-768.

Lei, D., Du, M., Wu, M., Chen, T., Huang, X., Du, X., . . \& \& Gong, Q. (2015). Functional MRI reveals different response inhibition between adults and children with ADHD. Neuropsychology.

Lijffijt, M., Kenemans, J. L., Verbaten, M. N., \& van Engeland, H. (2005). A meta-analytic review of stopping performance in attention-deficit/hyperactivity disorder: deficient inhibitory motor control? Journal of Abnormal Psychology, 114, 216-222.

Luna, B., \& Sweeney, J. A. (2004). The emergence of collaborative brain function: FMRI studies of the development of response inhibition. Annals of the New York Academy of Sciences, 1021, 296-309.

Martel, M. M., Roberts, B., Gremillion, M., von Eye, A., \& Nigg, J. T. (2011). External validation of bifactor model of ADHD: explaining heterogeneity in psychiatric comorbidity, cognitive control, and personality trait profiles within DSM-IV ADHD. Journal of Abnormal Child Psychology, 39, 1111-1123. doi:10.1007/s10802-011-9538-y.

McAuley, T., Crosbie, J., Charach, A., \& Schachar, R. (2014). The persistence of cognitive deficits in remitted and unremitted ADHD: a case for the state-independence of response inhibition. Journal of Child Psychology and Psychiatry, and Allied Disciplines, 55, 292300. doi:10.1111/jcpp.12160.

Meinzer, M. C., Pettit, J. W., \& Viswesvaran, C. (2014). The cooccurrence of attention-deficit/hyperactivity disorder and unipolar depression in children and adolescents: a meta-analytic review. Clinical Psychology Review, 34, 595-607. doi:10.1016/j.cpr.2014. 10.002

Merikangas, K. R., He, J. P., Burstein, M., Swanson, S. A., Avenevoli, S., Cui, L., . . \& \& Swendsen, J. (2010). Lifetime prevalence of mental disorders in U.S. adolescents: results from the national comorbidity survey replication-adolescent supplement (NCS-A). Journal of the American Academy of Child and Adolescent Psychiatry, 49, 980989. doi:10.1016/j.jaac.2010.05.017.

Nederhof, E., Ormel, J., \& Oldehinkel, A. J. (2014). Mismatch or cumulative stress: the pathway to depression is conditional on attention style. Psychological Science, 25, 684-692. doi:10.1177/ 0956797613513473.
Nigg, J. T. (2005). Neuropsychologic theory and findings in attentiondeficit/hyperactivity disorder: the state of the field and salient challenges for the coming decade. Biological Psychiatry, 57, 1424 1435.

Nigg, J. T., Willcutt, E. G., Doyle, A. E., \& Sonuga-Barke, E. J. (2005). Causal heterogeneity in attention-deficit/hyperactivity disorder: do we need neuropsychologically impaired subtypes? Biological Psychiatry, 57, 1224-1230.

Oldehinkel, A. J., Hartman, C. A., Van Oort, F. V., \& Nederhof, E. (2015a). Emotion recognition specialization and context-dependent risk of anxiety and depression in adolescents. Brain and Behavior, 5, e00299. doi:10.1002/brb3.299.

Oldehinkel, A. J., Rosmalen, J. G., Buitelaar, J. K., Hoek, H. W., Ormel, J., Raven, D., . . \& Hartman, C. A. (2015b). Cohort profile update: the TRacking adolescents' individual lives survey (TRAILS). International Journal of Epidemiology, 44(1), 76-76n. doi:10. 1093/ije/dyu225.

Ormel, J., Oldehinkel, A. J., Sijtsema, J., van Oort, F., Raven, D., Veenstra, R., . . \& \& Verhulst, F. C. (2012). The TRacking adolescents' individual lives survey (TRAILS): design, current status, and selected findings. Journal of the American Academy of Child and Adolescent Psychiatry, 51, 1020-1036. doi:10.1016/j.jaac.2012.08. 004.

Pauli-Pott, U., Dalir, S., Mingebach, T., Roller, A., \& Becker, K. (2014). Attention deficit/hyperactivity and comorbid symptoms in preschoolers: differences between subgroups in neuropsychological basic deficits. Child Neuropsychology, 20, 230-244.

Privitera, G. J., Agnello, J. E., Walters, S. A., \& Bender, S. L. (2015). Randomized feedback about diagnosis influences statistical and clinical significance of self-report ADHD assessment in adults. Journal of Attention Disorders, 19(5), 447-451.

Raiker, J. S., Rapport, M. D., Kofler, M. J., \& Sarver, D. E. (2012). Objectively-measured impulsivity and attention-deficit/hyperactivity disorder (ADHD): testing competing predictions from the working memory and behavioral inhibition models of ADHD. Journal of Abnormal Child Psychology, 40, 699-713.

Rajendran, K., Trampush, J. W., Rindskopf, D., Marks, D. J., O’Neill, S., \& Halperin, J. M. (2013). Association between variation in neuropsychological development and trajectory of ADHD severity in early childhood. American Journal of Psychiatry, 170, 1205-1211.

Rapport, M. D., Chung, K. M., Shore, G., Denney, C. B., \& Isaacs, P. (2000). Upgrading the science and technology of assessment and diagnosis: laboratory and clinic-based assessment of children with ADHD. Journal of Clinical Child Psychology, 29(4), 555-568.

Rapport, M. D., Orban, S. A., Kofler, M. J., \& Friedman, L. M. (2013). Do programs designed to train working memory, other executive functions, and attention benefit children with ADHD? A metaanalytic review of cognitive, academic, and behavioral outcomes. Clinical Psychology Review, 33, 1237-1252.

Rhodes, S. M., Park, J., Seth, S., \& Coghill, D. R. (2012). A comprehensive investigation of memory impairment in attention deficit hyperactivity disorder and oppositional defiant disorder. Journal of Child Psychology and Psychiatry, and Allied Disciplines, 53, 128-137. doi:10.1111/j.1469-7610.2011.02436.x.

Rommelse, N. N., Altink, M. E., de Sonneville, L. M., Buschgens, C. J., Buitelaar, J., Oosterlaan, J., \& Sergeant, J. A. (2007). Are motor inhibition and cognitive flexibility dead ends in ADHD? Journal of Abnormal Child Psychology, 35, 957-967. doi:10.1007/s10802007-9146-z.

Roy, A., Oldehinkel, A. J., Verhulst, F. C., Ormel, J., \& Hartman, C. A. (2014). Anxiety and disruptive behavior mediate pathways from attention-deficit/hyperactivity disorder to depression. The Journal of Clinical Psychiatry, 75, e108-e113.

Roy, A., Hartman, C. A., Veenstra, R., \& Oldehinkel, A. J. (2015). Peer dislike and victimisation in pathways from ADHD symptoms to depression. European Child \& Adolescent Psychiatry, 24, 887-895. 
Sebastian, A., Jung, P., Krause-Utz, A., Lieb, K., Schmahl, C., \& Tuscher, O. (2014). Frontal dysfunctions of impulse control - a systematic review in borderline personality disorder and attention-deficit/hyperactivity disorder. Frontiers in Human Neuroscience, 8, 698. doi:10.3389/fnhum.2014.00698.

Sergeant, J. A., Geurts, H., \& Oosterlaan, J. (2002). How specific is a deficit of executive functioning for attention-deficit/hyperactivity disorder? Behavioural Brain Research, 130, 3-28.

Sergeant, J. A., Geurts, H., Huijbregts, S., Scheres, A., \& Oosterlaan, J. (2003). The top and the bottom of ADHD: a neuropsychological perspective. Neuroscience \& Biobehavioral Reviews, 27, 583-592.

Shaw, P., Lerch, J., Greenstein, D., Sharp, W., Clasen, L., Evans, A., . . \& \& Rapoport, J. (2006). Longitudinal mapping of cortical thickness and clinical outcome in children and adolescents with attention-deficit/ hyperactivity disorder. Archives of General Psychiatry, 63, 540549

Shaw, P., Eckstrand, K., Sharp, W., Blumenthal, J., Lerch, J., Greenstein, D., . . \& Rapoport, J. (2007). Attention-deficit/hyperactivity disorder is characterized by a delay in cortical maturation. Proceedings of the National Academy of Sciences, 104, 19649-19654.

Shipstead, Z., Redick, T. S., Hicks, K. L., \& Engle, R. W. (2012). The scope and control of attention as separate aspects of working memory. Memory, 20, 608-628.

Sibley, M. H., Pelham Jr, W. E., Molina, B. S., Gnagy, E. M., Waxmonsky, J. G., Waschbusch, D. A., ... \& Kuriyan, A. B. (2012). When diagnosing ADHD in young adults emphasize informant reports, DSM items, and impairment. Journal of Consulting and Clinical Psychology, 80, 1052.

Sjowall, D., Roth, L., Lindqvist, S., \& Thorell, L. B. (2013). Multiple deficits in ADHD: executive dysfunction, delay aversion, reaction time variability, and emotional deficits. Journal of Child Psychology and Psychiatry, and Allied Disciplines, 54, 619-627. doi:10.1111/ jcpp.12006.

Slaats-Willemse, D., Swaab-Barneveld, H., De Sonneville, L., Van Der Meulen, E., \& Buitelaar, J. (2003). Deficient response inhibition as a cognitive endophenotype of ADHD. Journal of the American Academy of Child and Adolescent Psychiatry, 42, 1242-1248.

Snyder, H. R. (2013). Major depressive disorder is associated with broad impairments on neuropsychological measures of executive function: a meta-analysis and review. Psychological Bulletin, 139, 81.
Sonuga-Barke, E. J. (2003). The dual pathway model of AD/HD: an elaboration of neuro-developmental characteristics. Neuroscience \& Biobehavioral Reviews, 27, 593-604.

Stevens, J. P. (2009). Applied multivariate statistics for the social sciences. Taylor \& Francis.

Thissen, A. J., Rommelse, N. N., Hoekstra, P. J., Hartman, C., Heslenfeld, D., Luman, M., . . . \& Buitelaar, J. K. (2014). Attention deficit hyperactivity disorder (ADHD) and executive functioning in affected and unaffected adolescents and their parents: Challenging the endophenotype construct. Psychological Medicine, 44, 881-892. doi:10.1017/S0033291713001153.

van Deurzen, P. A., Buitelaar, J. K., Brunnekreef, J. A., Ormel, J., Minderaa, R. B., Hartman, C. A., . . . \& Slaats-Willemse, D. I. (2012). Response time variability and response inhibition predict affective problems in adolescent girls, not in boys: the TRAILS study. European Child \& Adolescent Psychiatry, 21, 277-287.

van Lieshout, M., Luman, M., Buitelaar, J., Rommelse, N. N., \& Oosterlaan, J. (2013). Does neurocognitive functioning predict future or persistence of ADHD? A systematic review. Clinical Psychology Review, 33, 539-560. doi:10.1016/j.cpr.2013.02.003.

Vance, A., Ferrin, M., Winther, J., \& Gomez, R. (2013). Examination of spatial working memory performance in children and adolescents with attention deficit hyperactivity disorder, combined type (ADHD-CT) and anxiety. Journal of Abnormal Child Psychology, 41, 891-900. doi:10.1007/s10802-013-9721-4.

Vloet, T. D., Konrad, K., Herpertz-Dahlmann, B., Polier, G. G., \& Gunther, T. (2010). Impact of anxiety disorders on attentional functions in children with ADHD. Journal of Affective Disorders, 124, 283-290. doi:10.1016/j.jad.2009.11.017.

Willcutt, E. G., Pennington, B. F., Boada, R., Ogline, J. S., Tunick, R. A., Chhabildas, N. A., \& Olson, R. K. (2001). A comparison of the cognitive deficits in reading disability and attention-deficit/hyperactivity disorder. Journal of Abnormal Psychology, 110, 157.

Willcutt, E. G., Doyle, A. E., Nigg, J. T., Faraone, S. V., \& Pennington, B. F. (2005). Validity of the executive function theory of attentiondeficit/hyperactivity disorder: a meta-analytic review. Biological Psychiatry, 57, 1336-1346.

Wittchen, H. U. (1994). Reliability and validity studies of the WHOcomposite international diagnostic interview (CIDI): a critical review. Journal of Psychiatric Research, 28, 57-84. 